\title{
What was Learned in Predicting Slender Airframe Aerodynamics with the F16-XL Aircraft
}

\author{
Arthur Rizzi ${ }^{1}$ \\ Royal Institute of Technology (KTH), Stockholm, Sweden \\ James M. Luckring ${ }^{2}$ \\ NASA Langley Research Center, Hampton VA, 23681, USA
}

The CAWAPI-2 coordinated project has been underway to improve CFD predictions of slender airframe aerodynamics. The work is focused on two flow conditions and leverages a unique flight data set obtained with the F-16XL aircraft for comparison and verification. These conditions, a low-speed high angle-of-attack case and a transonic low angle-of-attack case, were selected from a prior prediction campaign wherein the CFD failed to provide acceptable results. In re-visiting these two cases, approaches for improved results include better, denser grids using more grid adaptation to local flow features as well as unsteady higher-fidelity physical modeling like hybrid RANS/URANS-LES methods. The work embodies predictions from multiple numerical formulations that are contributed from multiple organizations where some authors investigate other possible factors that could explain the discrepancies in agreement, e.g. effects due to deflected control surfaces during the flight tests, as well as static aeroelastic deflection of the outer wing. This paper presents the synthesis of all the results and findings and draws some conclusions that lead to an improved understanding of the underlying flow physics, and finally making the connections between the physics and aircraft features.

\section{Contents}

I. Introduction/Background -Re-visit two CAWAPI cases ........................................................... 2

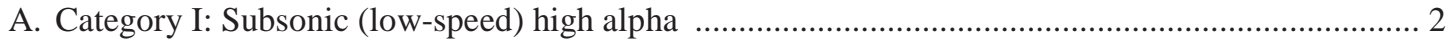

B. Category II: Transonic (high-speed) low alpha .............................................................. 3

II. Improved Physical \& Numerical Modeling ............................................................................. 4

A. Criteria for Assessment ................................................................................................... 4

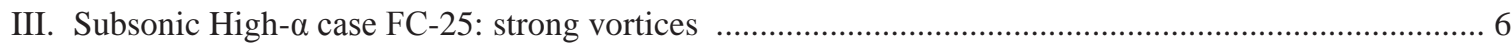

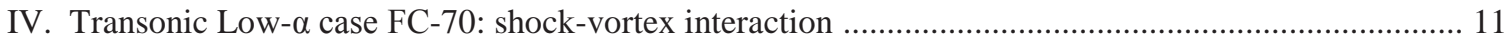

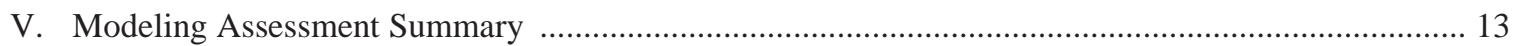

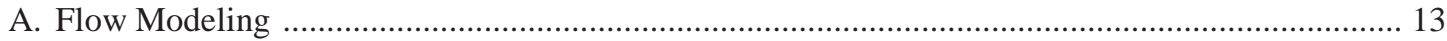

1. Better Grids

2. Improved Physics

B. Aircraft Modeling

1. Aeroelastics

2. Control Surfaces

VI. Concluding Remarks

\footnotetext{
${ }^{1}$ Professor, Department of Aeronautical \& Vehicle Engineering, AIAA Associate Fellow.

${ }^{2}$ Senior Research Engineer, Configuration Aerodynamics Branch, Mail Stop 499, AIAA Associate Fellow.
} 


\section{Nomenclature}

\begin{tabular}{|c|c|c|c|}
\hline $\begin{array}{l}\mathrm{BL} \\
\mathrm{C}_{\mathrm{n}}\end{array}$ & $\begin{array}{l}\text { Airplane butt line, positive starboard } \\
\text { pressure coefficient }\end{array}$ & $\begin{array}{l}\mathrm{R}_{\text {cref }} \\
\mathrm{U}\end{array}$ & $\begin{array}{l}\text { Reynolds number based on } \mathrm{C}_{\mathrm{ref}}, \mathrm{U}_{\mathrm{ref}} / \mathrm{V} \\
\text { free stream reference velocity }\end{array}$ \\
\hline CS & Control surface & $\mathrm{x}, \mathrm{y}, \mathrm{z}$ & body-axis Cartesian coordinates \\
\hline c, Clocal & wing chord & $\alpha$ & angle of attack, deg. \\
\hline $\mathrm{C}_{\mathrm{ref}}$ & reference chord & $\beta$ & angle of sideslip, deg. \\
\hline FC & Flight Condition & $\mu$ & viscosity \\
\hline FS & Airplane fuselage station, positive aft & $v$ & kinematic viscosity, $\mu / \rho$ \\
\hline M & Mach number & $\rho$ & density \\
\hline
\end{tabular}

Organizations and Acronyms

$\begin{array}{ll}\text { A-D\&S } & \text { Airbus, Defense and Space, Germany } \\ \text { CAWAPI } & \text { Cranked Arrow Wing Aerodynamics Project, International (previous) } \\ \text { CAWAPI-2 } & \text { Cranked Arrow Wing Aerodynamics Project, International (current) } \\ \text { FOI } & \text { Swedish Defense Research Agency, Sweden } \\ \text { KTH } & \text { Royal Institute of Technology, Sweden } \\ \text { LaRC } & \text { Langley Research Center, USA } \\ \text { NASA } & \text { National Aeronautics and Space Administration, USA } \\ \text { NLR } & \text { National Aerospace Laboratory, Netherlands }\end{array}$

\section{Introduction/Background - Re-visit two CAWAPI cases}

The considerable progress that was achieved during the original CAWAPI to better predict the slender airframe 1 aerodynamics with the F16-XL aircraft is reported in references [1-5] from a special edition of the AIAA Journal of Aircraft. In addition to this progress, the collective CAWAPI team also identified two categories of flight conditions for which all the CFD failed to produce acceptable predictions [6]. The first category is low-speed, high angle-of-attack flight that is relevant to take-off and landing operations. The second is transonic, low angle-of-attack flight that is relevant to transonic cruise operations. While most of the CFD agreed fairly well among itself, correlations with the flight data were less than desired. Both categories have complex flow physics that can be very challenging to our current computational capabilities that provided the motivation to launch CAWAPI-2 to re-visit these two flight categories.

\section{A. Category I: Subsonic (low-speed) high alpha}

The subsonic high angle-of-attack case is dominated by multiple interacting vortical flows. Vortex-vortex interactions can be very difficult to compute, and at the subject conditions the flow is most likely unsteady. In addition, this condition may include vortex breakdown flow physics that could be exacerbated by the ogive contour of the leading edge at the apex of the wing. Nevertheless, predictions from the CAWAPI work were not too bad for the inboard portion of the wing, but were less than desired on the outboard portion where the vortical interactions and flow unsteadiness could be significant. Figure 1, taken from Morton ${ }^{6}$ [2007], illustrates the situation. These were the only unsteady simulations carried out in CAWAPI. The moderate alpha results, FC-07 at $\alpha=11^{\circ}$, proved to be in fact steady, and agreed well with the other steady CFD results as well as with flight test data. But when the angle of attach increases to $\alpha=20^{\circ}, \mathrm{FC}-25$, the authors reported their results to be unsteady, particularly on the outer wing. Their time-averaged values agreed better with the flight tests than any of the other steady CFD did, but the comparison was less than satisfactory. 

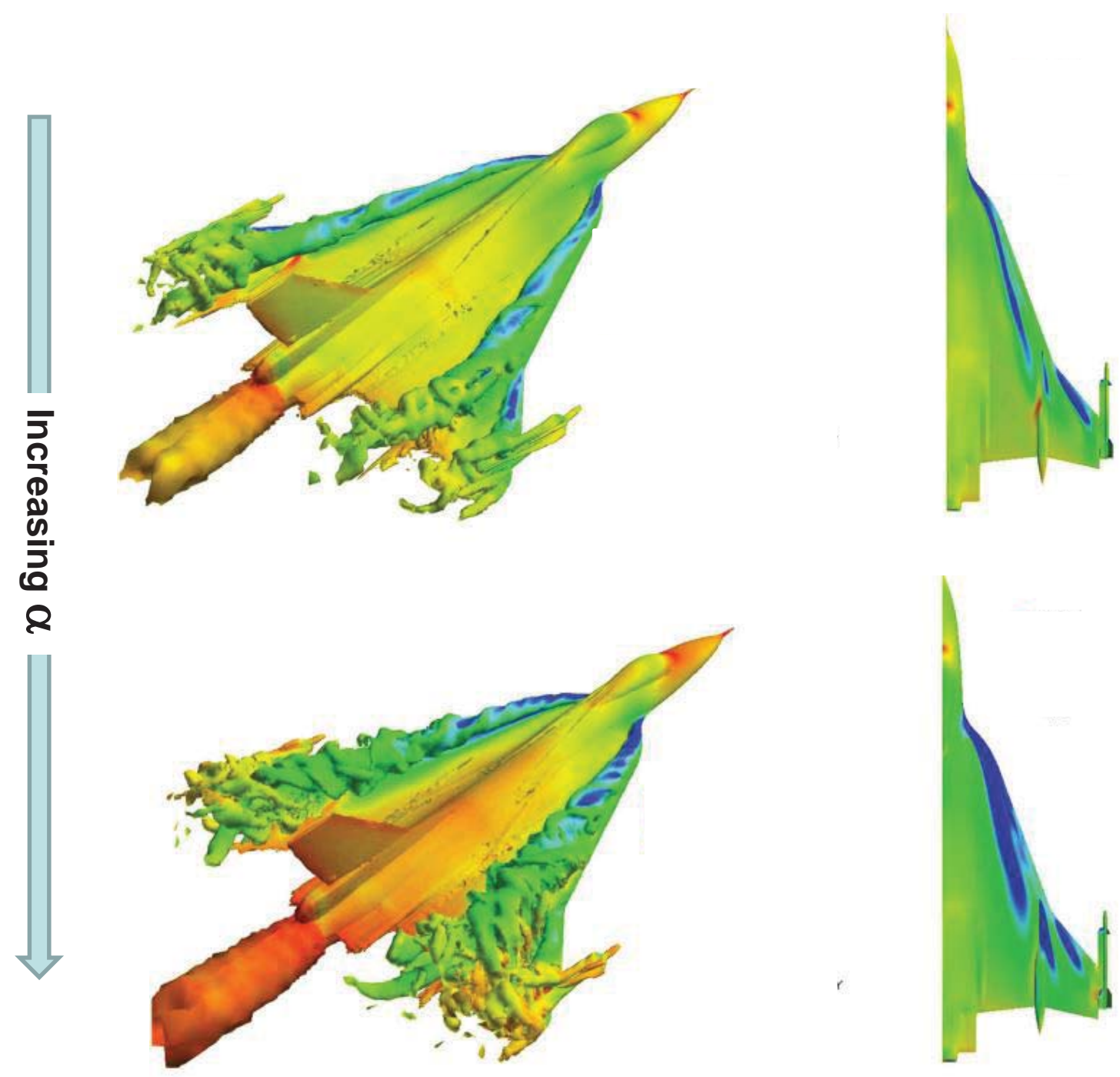

Figure 1. Effects of increasing alpha from FC-07 $\left(\alpha=11^{\circ}\right)$ to FC-25 $\left(\alpha=20^{\circ}\right)$ : Subsonic high angle-of-attack category. $M=0.24, R_{\text {cref }}=32 \times 10^{6}$.

\section{B. Category II: Transonic (high-speed) low alpha}

The transonic low angle-of-attack case FC-70 exhibits a series of longitudinal shocks as well as wing leadingedge vortices that lift out over the boundary layer despite the relatively low angle of attack, see Figure 2. For this case shock-vortex interactions will be among the critical flow physics requiring sufficient computational modeling. Finer scale shocks than those shown in the left part of Figure 2 could also be present in the vicinity of the leadingedge vortices at these transonic conditions. Such shocks can be difficult to resolve in flight-test measurements. All of the CFD predictions from the CAWAPI effort missed measured flow details on the inboard portion of the wing. This paper summarizes what has been learned in CAWAPI-2 by re-visiting these two flight categories, starting with a reassessment of the aircraft modeling and the flight data, an improved flow modeling and how that leads to an improved understanding of the underlying flow physics, and finally making the connections between the physics and aircraft features.

The convergence-divergence of skin-friction lines in Figure 2 show the confluence of vortical layers near the midspan of the wing that likely separates and reattaches inboard near the fuselage. Separation does seem to occur, but the liftoff appears to remain close to the upper surface, either just in or just above the boundary layer. The flow 
does separate from the air dam and actuator pod and a small vortex seems to develop. Note, however, that no vortex is shed from the outer wing and there is no sign of a secondary vortex over the inner wing, as found in the other cases. Iso-surfaces of constant Mach=1 values in the left in Figure 2 show the presence of three shock waves.

Highly adapted meshes are needed to resolve the interaction of the weak vortex with the relatively strong shock waves. Tomac ${ }^{7}$ [2014] has used a grid adaption technique in three steps. The first adaptation uses a gradient sensor to mainly seek out shock waves, the second adaptation uses the lamda2 sensor [Le Moigne ${ }^{8}, 2004$ ] that tracks vortex cores (as seen in Figure 2), and the third adaptation is again the gradient sensor.

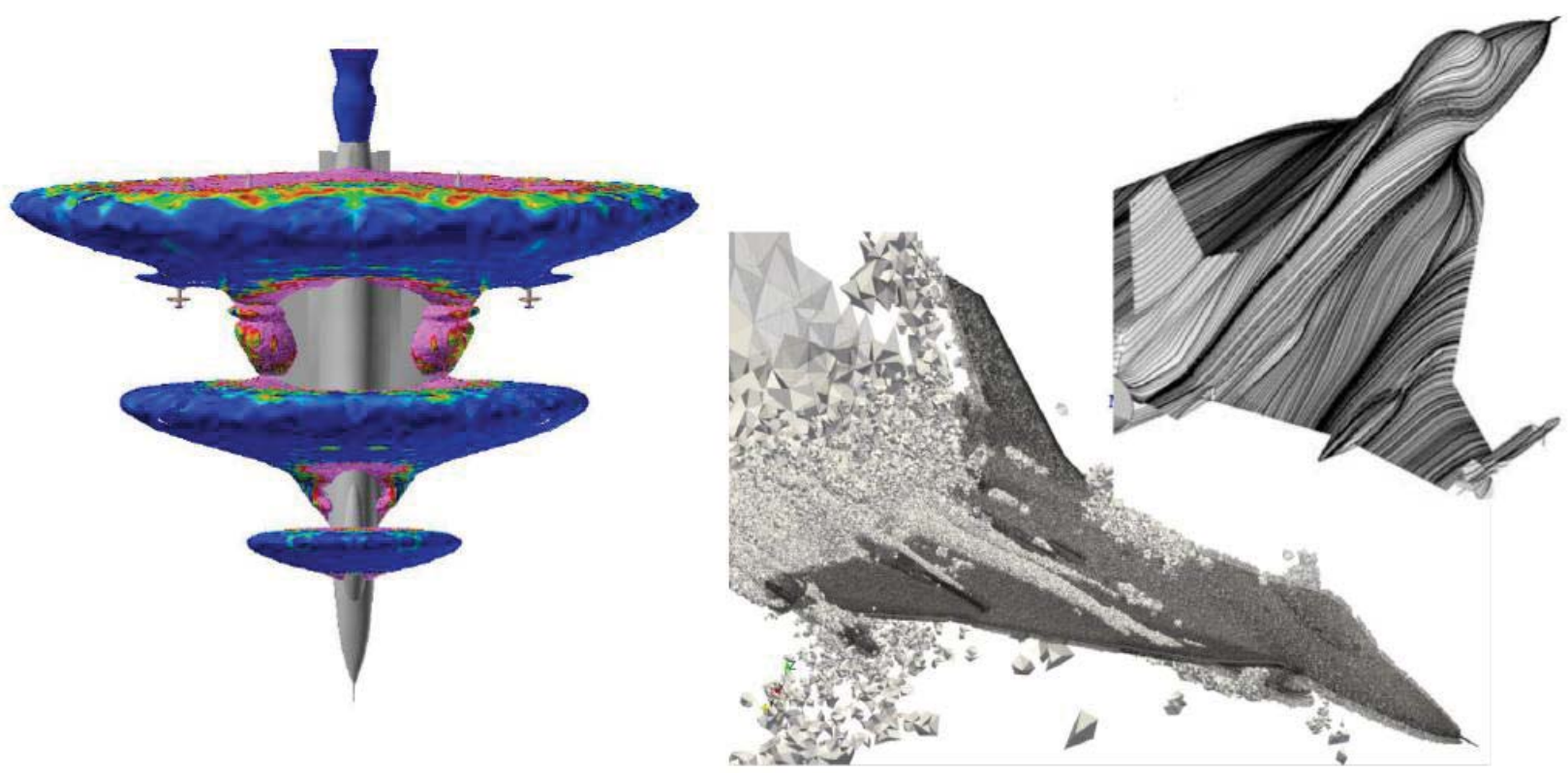

Figure 2. Transonic low angle-of-attack case where shocks are strong and vortices are fewer and weaker. FC-70, $M=0.97, R_{\text {cref }}=89 \times 10^{6}, \alpha=4^{0}$.

\section{Improved Physical and Numerical Modeling}

Among the improvements in the physical modeling is time-accurate unsteady simulation with either URANS modeling or hybrid URANS-LES modeling to capture the unsteady vortical flow features that are expected in FC25. Even the effect of different RANS turbulence modeling is assessed. In addition multi-physics phenomena like fluid-structure interaction is also modeled to account for possible deflections of the wing surface especially for the cases at high dynamic pressure and/or high lift loading. The geometrical configuration for all CAWAPI cases was with all control surfaces at zero deflection angle. The flight conditions of interest are for trimmed 1-g level flight, and although the surface deflection needed for trim in these cases are known to be small, there nevertheless could be some small surface deflections. And so control-surface deflection is modeled in order to gauge its effect.

Among the improvements in numerical models is foremost higher grid resolution obtained with denser overall grids than those used in CAWAPI, and adaptive grid refinement.

\section{A. Criteria for Assessment}

The primary criteria for assessing the correctness of the computational results is the comparison of computed surface pressure with those that were measured in flight. The measurements were taken along butt lines and fuselage-station lines, as shown in Figure 3. 


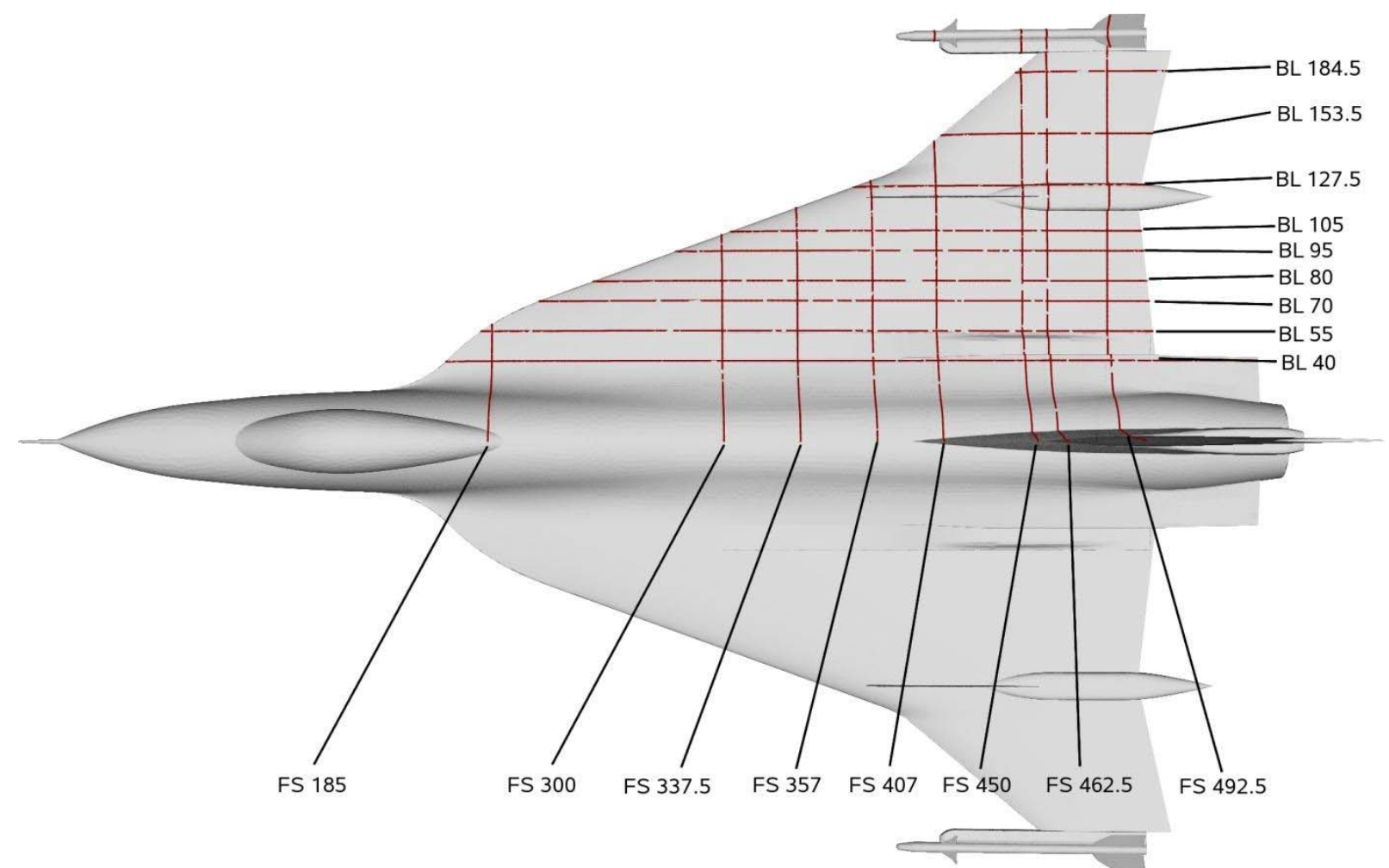

Figure 3. F-16XL spanwise Fuselage Stations and chordwise Butt Lines along which surface pressures were measured.

The flight conditions chosen for CAWAPI-2 investigations are shown in Figure 4 along with those from the predecessor research project, CAWAPI. The detailed CAWAPI flight conditions at moderate angles of attack and moderate Mach numbers have been omitted for clarity. These conditions were fairly well predicted form the CAWAPI project.

The CAWAPI-2 conditions were chosen primarily to help establish trends in the measured pressures associated with the vortex and shock structures. In addition some flight conditions were chosen to help address some uncertainties associated with the flight data. At transonic speeds the cases were chosen to help address control surface deflection uncertainty [see Boelens ${ }^{11}$, 2014]. At low speeds the cases were chosen from independent flights at nearby flight conditions such that the results should be aerodynamically similar [see Hitzel ${ }^{12}$, 2014].

By virtue of working with flighttest data, it is also worth recalling that the data are at high (full-scale) Reynolds numbers for an operational

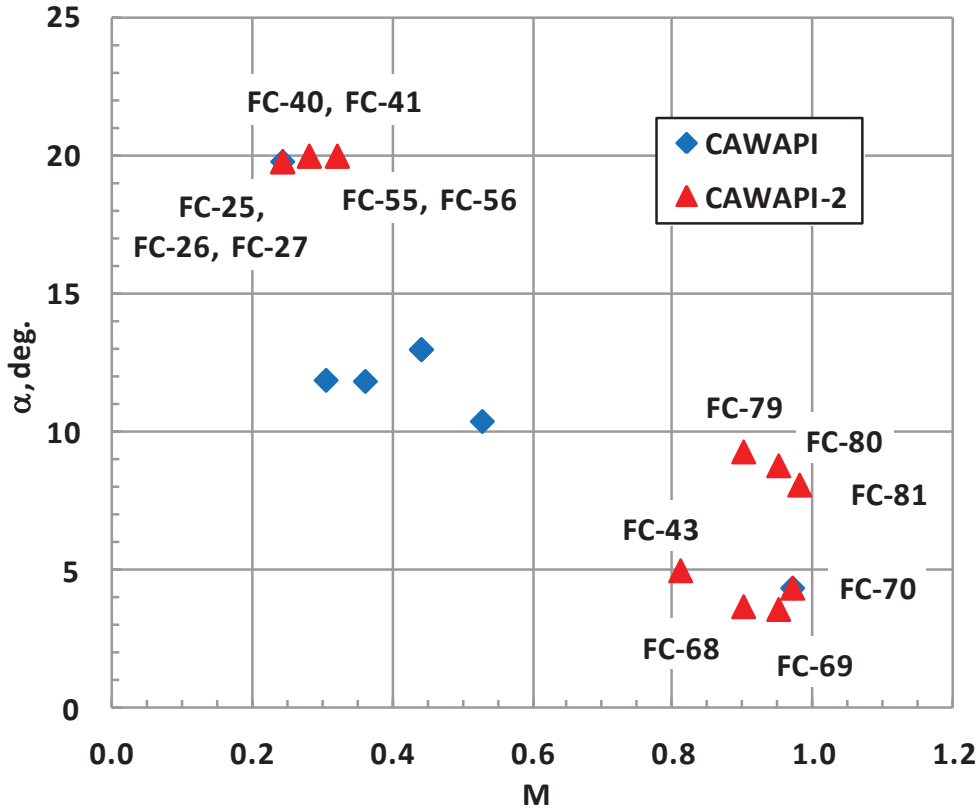

Figure 4. Mach- $\alpha$ parameter space of the flight conditions treated in CAWAPI and CAWAPI-2. aircraft geometry with all its corresponding complexities. 


\section{Subsonic High-Alpha Case FC-25: Strong Vortices}

The subsonic high angle-of-attack work for CAWAPI-2 was focused on the outer, $50^{\circ}$ swept wing panel aerodynamics. Inboard aerodynamics were fairly well predicted from the previous CAWAPI program. Some details for the challenging outer panel flowfield need to be first established.

Multiple complex flow phenomena occur and interact over this portion of the wing. The outer panel has three primary vortices in close proximity, one from the $70^{\circ}$ swept inboard leading edge that will persist over the outboard wing, one from the $50^{\circ}$ outboard leading edge, and a counter-rotating primary vortex from the air dam. Each of these vortices will also induce secondary vortices. An example is shown in Figure 5 from the new CAWAPI-2 work of the National Aerospace Laboratory, NLR Thus, a highly complex vortex-interaction flowfield will exist over the wing. Moreover, the sweep of the outboard panel in conjunction with the angle of attack for this case will most likely result vortex breakdown occurring in the nearfield of the wing and among the interacting vortices. Vortex breakdown is an inherently unsteady phenomena, so unsteady flow effects are likely important to the outer panel. It is worth noting that neither vortex interaction nor vortex breakdown flows are well predicted, and thus the interaction of these flows on aircraft geometry presents a daunting task to computational aerodynamics.

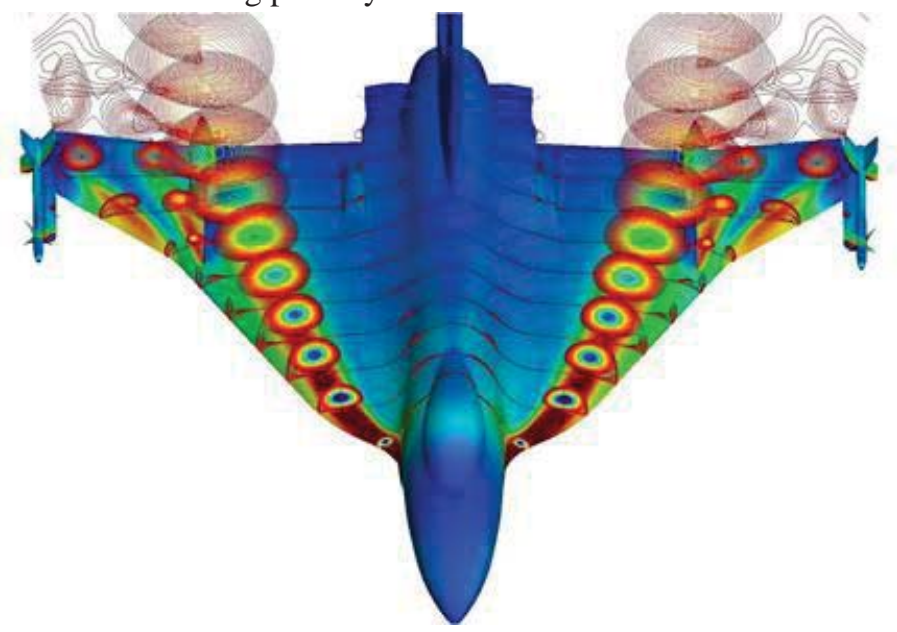

Figure 5. Subsonic high angle-of-attack case. FC-25, $M=0.24, R_{\text {cref }}=32 \times 10^{6}, \alpha=20^{\circ}$.

A few aspects of the outer panel geometry are also noteworthy. First, the outer panel has both leading-edge and trailing-edge control surfaces, Figure 6. Control surfaces have small longitudinal gaps between the surface and the neighboring wing, and these gap effects can affect vortical flows as was shown by Boelens $^{9}$ [1995] for the X-31 aircraft. No gaps were modeled in the predecessor CAWAPI work, and moreover all control surface deflections were assumed to be zero for CAWAPI. These surfaces constitute a large portion of the outer panel, and even small deflections could have important effects on the outboard flow. Possible control surface deflection effects for the flight data were reexamined as part of CAWAPI-2 although this work was chiefly focused on the transonic case. As a second aspect, the outer panel could experience aeroelastic effects due

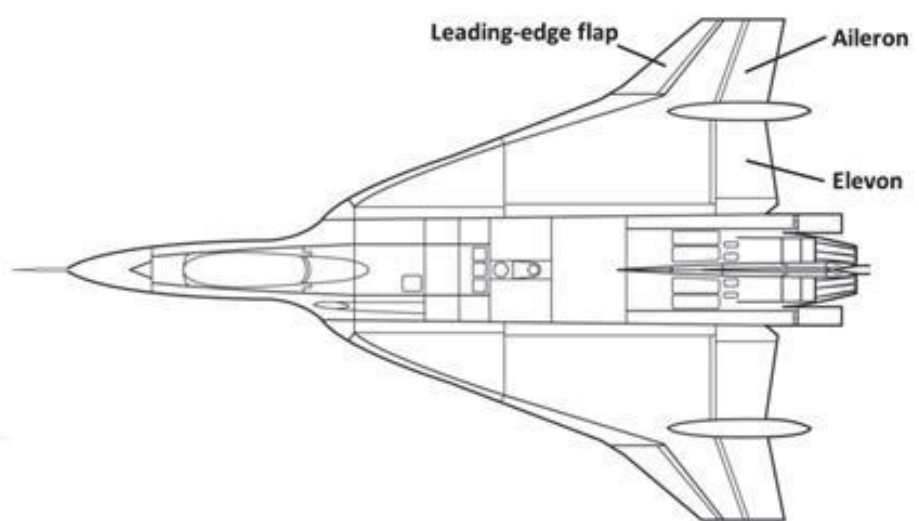

Figure 6. F-16XL control surfaces. to the thin geometry as well as the heavy loading at high angle of attack.

One of the first things the CAWAPI-2 team chose to do was a reexamination of the flight data set reported by Lamar $^{10}$ [2001] for additional flight data that could lend insight to the focus problems in basically either of two ways. First, conditions were sought that in some manner would modulate the particular flow physics of interest as manifested on the aircraft. Second, nearby conditions were sought that would exhibit similar flows but from different flights to address some of the vagaries inherent to flight data. It is common sense to leverage an existing data set (and especially flight data), but this physics-based approach to seeking further understanding of such complex flows is commendable. 
For the subsonic case, this led to the expanded conditions shown in Table 1, and much of the reasoning here was developed by Hitzel ${ }^{12}$ [2014] from a vortex breakdown perspective. FC-26 and FC-27 match FC-25 conditions except for positive/negative sideslip. Vortex strength, trajectory, and breakdown effects will be modulated from a simple-sweep perspective and thus provide some anticipated trends. The flight condition pairs FC-40/FC-41 and FC-55/FC-56 provide baseline and sideslip effects at slightly elevated Mach numbers. The Mach effect would be expected to be small and likely predictable, so these data would also add some confidence to the interpretation of the in-flight measurements. Periodic revisiting of the dataset occurred as the CAWAPI-2 work progressed. A philosophically similar approach to the transonic case will be reported in the next section.

All of the improved physical and numerical modeling capabilities discussed in the preceding section will pertain to this challenge problem, and in one way or another are they all coupled together. For example, these vortical interacting flows have many length scales, and the effects of grid resolution will not only impact turbulence model effects as regards wall-bounded flows, but also vortex interaction effects which can include inviscid-like free-field vorticity dynamics. Three CAWAPI-2 partners contributed to the subsonic high-alpha case, and each partner's focus included different subclasses of the aggregate challenges associated with the flow. Some highlights of their work follow, and each author included more work than could be reported in this paper.

A particular focus on vortex breakdown was reported by Hitzel $^{12}$ [2014] who based his work on manually adapted unstructured grids with 45 million points on the semispan. Two adaptations were developed, and one example is shown in Figure 7. Targeted clustering was developed for the regions where vortices were anticipated, and these grids were much finer than those previously used in the CAWAPI work even though the total grid size was only approximately doubled. Aircraft modeling was improved, and Hitzel reported that his surface geometry was developed without any geometric compromise or simplification. Baseline results with a RANS-k- $\omega$ flow model showed good overall agreement with FC-25 and other turbulence models were assessed. The CAWAPI-2 grids generally showed crisper vortex resolution, especially on the outer panel, than either the manual or solution adapted CAWAPI grid results.

New steady flow simulations were shown for the two sideslip cases, FC-26 and FC-27. At sideslip conditions, the windward vortices will strengthen while the leeward vortices will weaken compared to the no sideslip case. This also means that the tendencies for a vortex to breakdown will be increased on the windward side and decreased on the leeward side.

Hitzel's correlations with flight test for the inboard $70^{\circ}$-swept panel were generally good. Here the vortices are not burst. The windward outer panel was heavily burst in this simulation, but the simulation also indicated that vortex breakdown was absent on the leeward outer panel. Correlations with experiment looked better on the leeward panel where the computed vortex was coherent than they did for the windward outer-panel where vortices were clearly broken down. This implies that vortex breakdown is a likely contributor to the complexities of the outer panel flow. His analysis also indicates that the zero sideslip case (FC-25) may be an incipient breakdown condition. Incipient flows are perhaps the most difficult to predict and can be very sensitive to small changes (e.g., approaching flow, geometry, etc.). 
Unsteady sideslip analysis was then performed with a URANS-SAS formulation and demonstrated unsteady effects on the aft part of the inner wings and on the outboard panel and these unsteady effects were attributed to vortex breakdown. An example is shown in Figure 8 with snapshots at two times from the timeaccurate simulation. Pressure comparisons with experiment were shown for the two time snapshots as well as for time-averaged results. The overall correlation of the timeaveraged pressures with experiment was encouraging, and one example is shown in Figure 9 for the outer-most Butt Line where, in general, the CAWAPI predictions were insufficient. This result further indicates that unsteady effects largely associated with vortex breakdown flow physics could be important to the outer panel aerodynamics.

Hitzel also observed that the flow properties in the core of the inner panel leading-edge vortex were very peculiar for such a highly swept wing. At subsonic Mach numbers slender wings typically have a jet-like flow (velocity excess) down the vortex core, but for the F-16XL the core had a wake-like (velocity deficit) flow down the core. Such details of the core flow in a leading edge vortex can be significant to vortex breakdown. His analysis connected this vortex peculiarity to the s-blend portion of the leading edge that interfaces the wing to the fuselage. The s-blend was created during the F-16XL configuration development to increase high angle-ofattack stability, and Hitzel's analysis now connects this configuration development feature to a fundamental alteration of the inner leading edge vortex. Details can be found in his paper [Hitzel ${ }^{12}$, 2014].

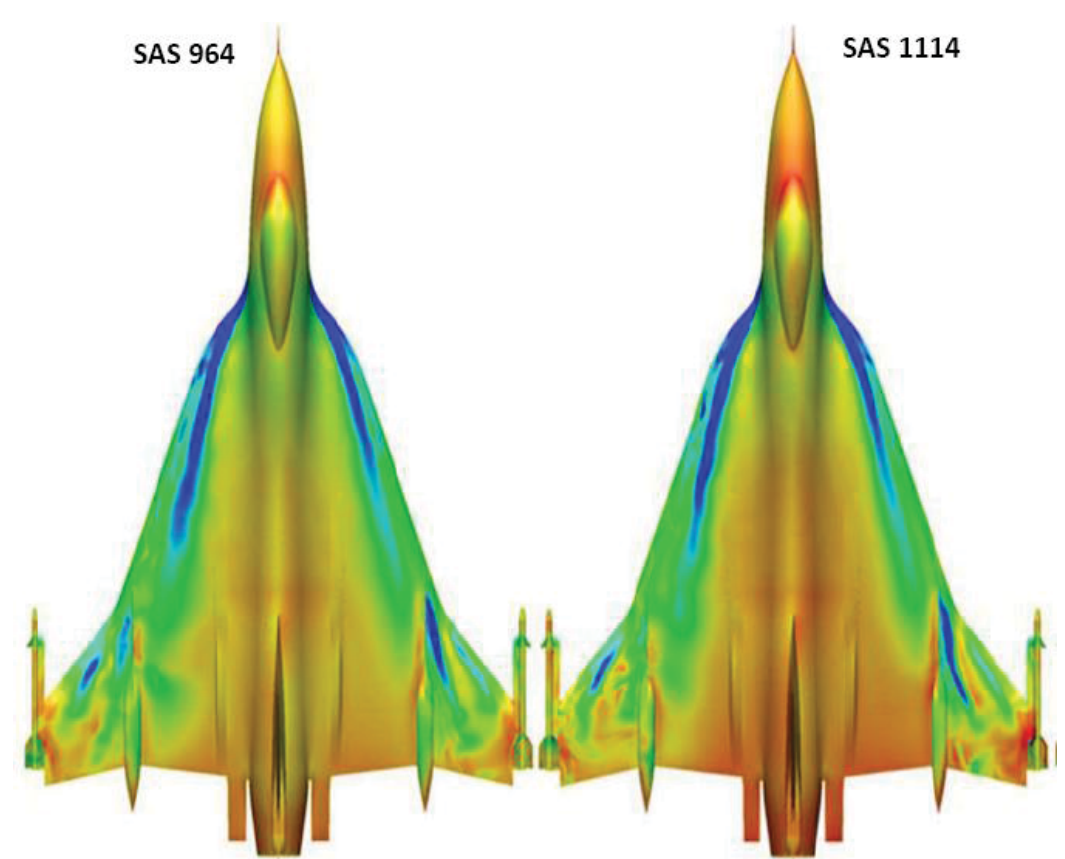

Figure 8. Unsteady sideslip predictions. $M=0.24, R_{\text {cref }}=32 \times 10^{6}$, $\alpha=20^{\circ}, \beta=-5^{0}$. From Hitzel ${ }^{12}$ [2014].

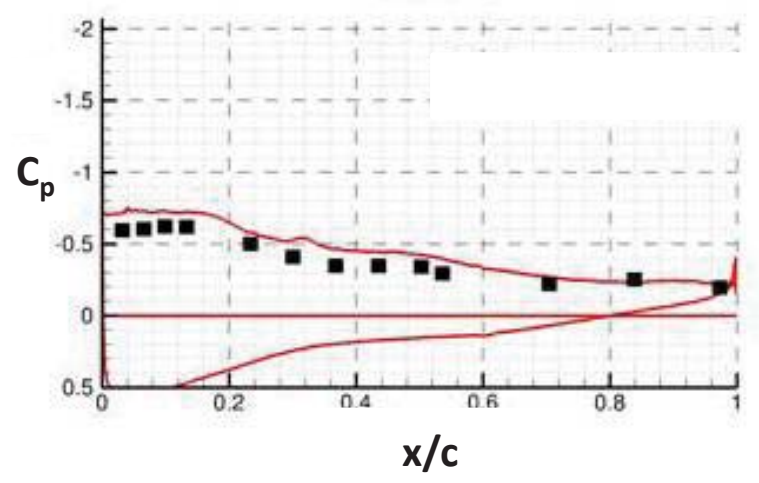

a) Windward outer panel

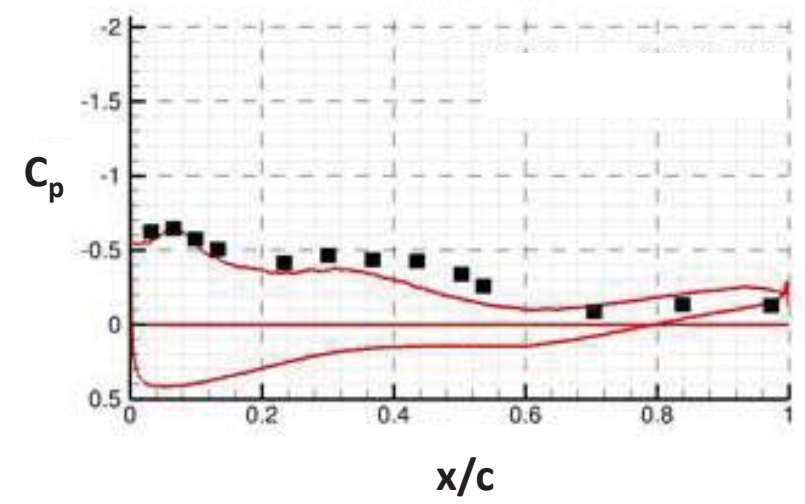

b) Leeward outer panel

Figure 9. Time-averaged pressures, Butt Line 184.5. FC-26/27, $M=0.24, R_{\text {cref }}=32 \times 10^{6}, \alpha=20^{\circ}, \beta=+/-5^{\circ}$. From Hitzel ${ }^{12}$ [2014]. 
Elmiligui $^{13}$ [2014] also performed unstructured computations and included grid resolution effects, turbulence model effects, and some applications of an adaptive grid technology based on numerical error estimation. These were the finest grids of all the CAWAPI-2; they included 19.4 million, 62.5 million, and 143.0 million cells, and a grid sensitivity study followed guidelines recently developed through the drag prediction and high-lift prediction workshops.

The medium grid $(62.5 \mathrm{M})$ was a baseline, and turbulence model assessments showed an unanticipated improvement on the inboard $70^{\circ}$-swept panel, up near the s-blend, from the $\mathrm{k}-\varepsilon$ model by providing improved simulation of the inboard panel secondary vortex. See Figure 10. When combined with the finest grid, the k- $\varepsilon$ model appeared to produce good simulations of pressures generally associated with a secondary vortex near the leading edge. An example for Butt Line 70 is shown in Figure 11. Although the focus of this work was the outer panel flow, the lower dissipation of the $k-\varepsilon$ model, coupled with the much finer grids appears to have produced this improved modeling of the secondary vortical flow. Predictions near the tip of the outer panel were still not improved by grids or turbulence models, thus implying that other physics, such as unsteady flows, may be important there.

Error estimation technology was used to show error bands on predicted pressure distributions in the context of grid refinement studies. Pressure error bars estimated from a coarse grid solution were shown to contain, for the most part, the pressure estimates from the medium grid solution. Then pressure error bars estimated from the medium grid solution were shown to contain, for the most part, the pressure estimates from the fine grid solution. The method seems to provide a means to estimate errors associated with further but unperformed grid resolution. This would apply only to circumstances where the physics are established and only being further resolved by the grid refinements, (i.e., no new physics are being manifested through the grid refinement process).

The adaptive grid resolution study showed very little effect on the results, and this was somewhat surprising. Only one case, FC-25, was studied, and only limited analysis was shown. Further assessments of the adaptive approach would be warranted

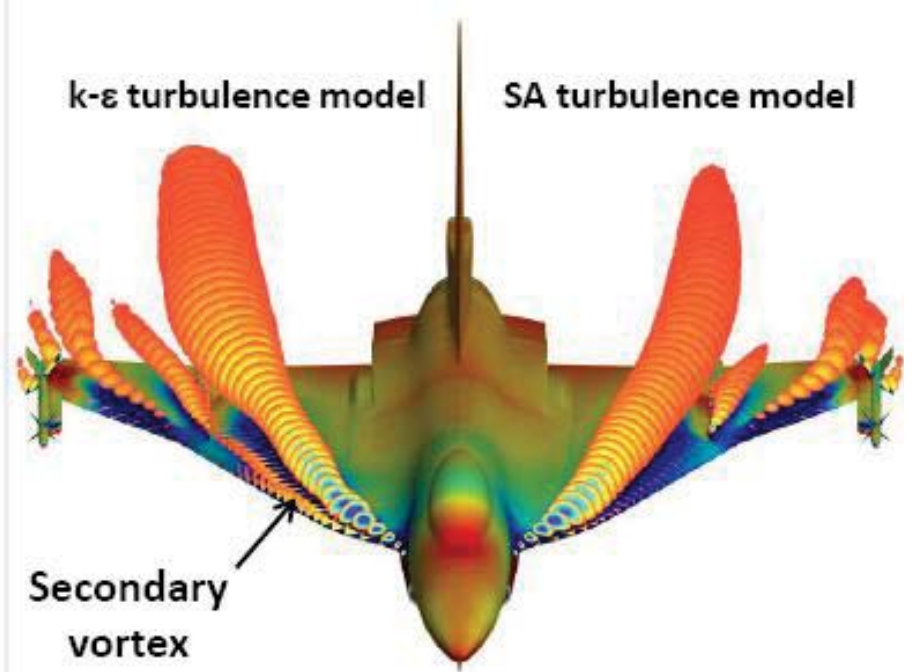

Figure 10. Secondary vortex modeling. $\mathrm{F} C \mathrm{C}-25, \mathrm{M}=\mathbf{0 . 2 4}$, $\mathbf{R}_{\text {cref }}=32 \times 10^{6}, \alpha=20^{\circ}$. From Elmiligui ${ }^{13}$ [2014].

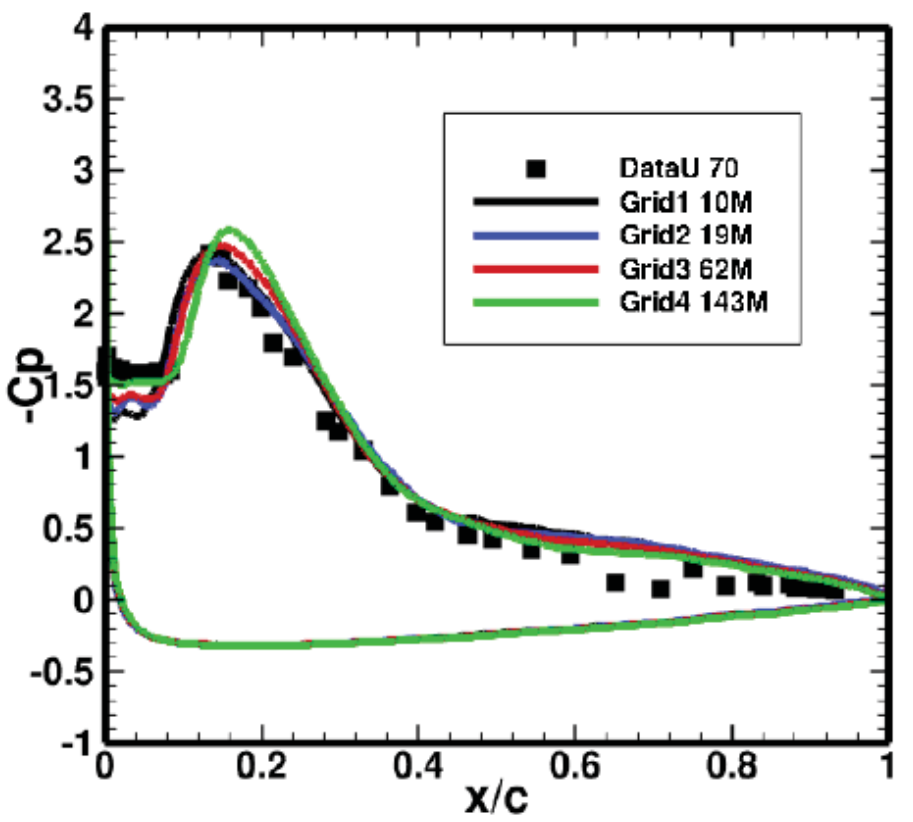

Figure 11. Pressure distribution, Butt Line 70, k- $\varepsilon$ turbulence model. FC-25, $M=0.24, R_{\text {cref }}=32 \times 10^{6}, \alpha=20^{\circ}$. From Elmiligui ${ }^{13}$ [2014]. 
Unique contributions for low-speed control surface and static aeroelastic deformation effects were reported by Jirasek ${ }^{14}$ [2014]. The work was unstructured and included steady RANS and unsteady URANS simulations with an EARSM turbulence model. An example of the control surface gridding is shown in Figure 12, and this modeling accounted for the gaps between the control surfaces and the wing. A simple first-order, plate-based aeroelastic formulation was used for this work. Details of the formulation can be found in the transonic application study, also by Jirasek ${ }^{15}$ [2014], which used this same modeling.

A rigid-wing application with the control surface gaps but no control surface deflections was performed for the FC-25 case. Hitzel ${ }^{12}$ [2014] had demonstrated the importance of sideslip to this flight condition, with probable incipient vortex breakdown, and Jirasek modeled the full span configuration to include the actual FC-25 sideslip angle, $\beta=0.7^{\circ}$. Results are shown in Figure 13, and inspection of the outer $50^{\circ}$ swept panels clearly shows different vortical footprints. It thus appears that this actual sideslip value could be another factor important to the detailed comparisons of surface pressures between flight measurement and CFD.

Significant effects of static aeroelastic deflections as well as control surface deflections were found, and one example is shown in Figure 14 for BL 153 on the outer panel. Here the baseline solution (steady RANS, rigid, no deflections) is shown with the black solid line. The corresponding steady RANS aeroelastic solution (red dashed line) is notably in better agreement with the flight-test measurements over the entire chord. The figure also includes a contrast between steady and unsteady simulations for the rigid wing, and the unsteady flow simulation is also closer to experiment. The control surface effects on this figure were a sensitivity/demonstration assessment study; the deflection angles chosen do not necessarily correspond to FC-25 flight conditions.

There was a balanced suite of effects studied among the CAWAPI-2 team members for the low-speed high-angle-of-attack focus condition. Unsteady aerodynamics, vortex breakdown, and static aeroelastic effects all seem to be important to the outer panel flowfield, and significantly improved correlations with experiment have been demonstrated as compared to the conclusion of the CAWAPI program. Denser grids and targeted grid clustering have also contributed to these improved correlations.

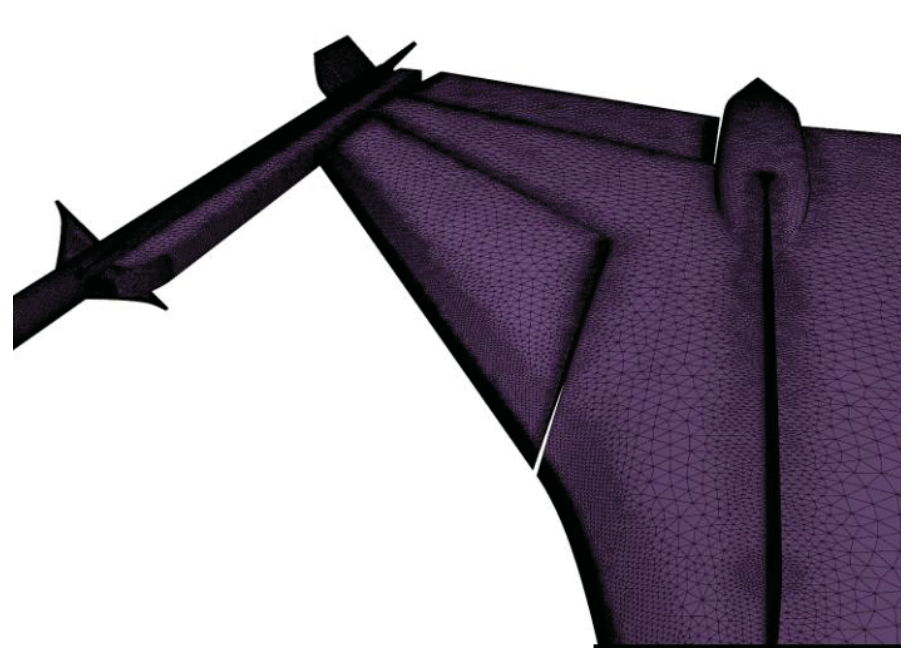

Figure 12. Control surface grid. From Jirasek ${ }^{14}$ [2014]

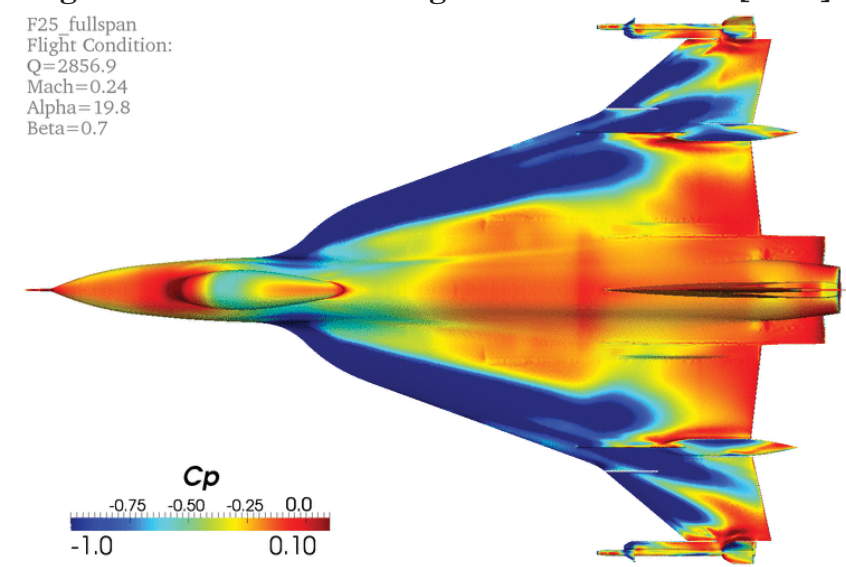

Figure 13. Full-span solution. FC-25, $M=0.24$, $\mathbf{R}_{\text {cref }}=32 \times 10^{6}, \alpha=20^{\circ}, \beta=0.7$. From Jirasek ${ }^{14}[2014]$

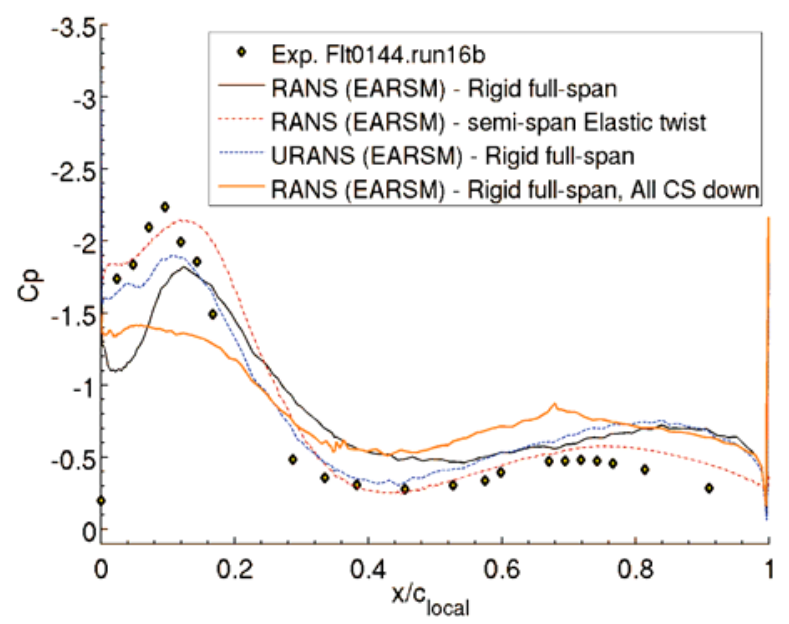

Figure 14. Chordwise outer panel pressures, BL-153.

$$
\text { FC-25, } M=0.24, R_{\text {cref }}=32 \times 10^{6}, \alpha=20^{\circ} \text {. }
$$

From Jirasek $^{14}$ [2014] 


\section{Transonic Low-Alpha Case FC-70: Shock-Vortex Interaction}

The most unsatisfactory comparisons presented in CAWAPI were those for FC-70. All of the CFD results agreed so well with each, and so poorly with the flight-test data, particularly in the inner-wing region [6]. This poor agreement instigated an effort, in the closing phase of CAWAPI to investigate possible causes for such discrepancy. Several authors tried better, denser grids, including adaptation, to improve the comparison but without success. It was conjectured that other factors could be responsible for discrepancy, among these, that some control surface (or surfaces) were deployed during the flight test and not modeled in the CFD, or that there were aeroelastic deflections of airframe (most likely on the outer wing panel)
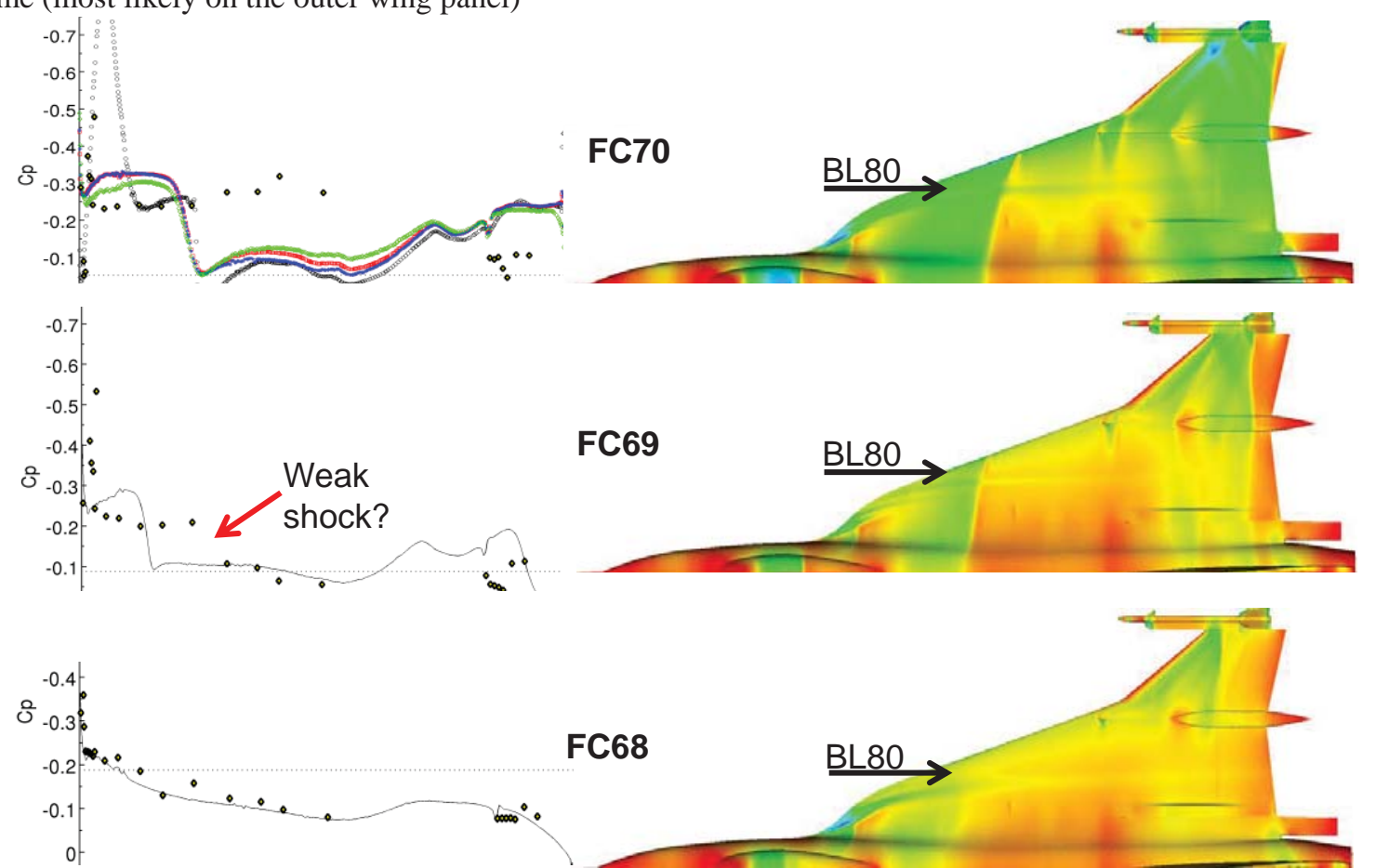

Figure 15. Comparison of three results with increasing Mach number, $M=0.90,0.95,0.97$ with the same incidence, $\alpha=4^{0}$. Flight Conditions FC-68, FC-69, FC-70. Steady simulations.

A major objective of CAWAPI-2 is to carry out a thorough examination of possible reasons for the discrepancy. As for the high alpha cases, the approach taken uses denser grids along with mesh adaption to relevant flow features, in particular the shocks along with the vortices. Where appropriate, unsteady modeling may also be investigated. But foremost to reaching an understanding about the flow physics involved is to set in place a systematic study of a sequence of flight conditions with increasing shock and vortex strengths, varying independently of each other. Figure 15 indicated how such sequences could be conceived.

Table 2 lists the expanded conditions for transonic flight available to us. Two sequences of increasing Mach number can be identified; one at low alpha FC68 -FC-69 -FC-70, and another at moderate alpha FC79 -FC-80 -FC-81. FC-43 can be selected as a lowalpha and low-Mach condition where transonic effects such as shock-wave-vortex interaction is minimal, and hence more like the low speed moderate condition of FC-07.

A. Mach Sweep at Low alpha: FC68 -FC69-FC70

The results of one such sequence, that of increasing shock strength at constant low alpha (i.e. constant, weak vortex) was shown in Figure 15,
Table 2. CAWAPI-2 transonic flight conditions, nominal values

\begin{tabular}{|c|c|c|c|c|}
\hline FC & $\mathbf{M}$ & $\mathbf{R}_{\text {cref }}$ & $\boldsymbol{\alpha}, \mathbf{d e g}$. & $\boldsymbol{\beta}, \mathbf{d e g}$. \\
\hline \multicolumn{5}{|c|}{ Initial Condition } \\
\hline 70 & 0.97 & $88.8 \times 10^{6}$ & 4.4 & 0 \\
\hline \multicolumn{5}{|c|}{ Expanded Conditions } \\
\hline 43 & 0.81 & $69.6 \times 10^{6}$ & 5 & 0 \\
\hline 68 & 0.90 & $88.8 \times 10^{6}$ & 3.7 & 0 \\
\hline 79 & 0.90 & $88.8 \times 10^{6}$ & 9.3 & 0 \\
\hline 69 & 0.94 & $88.8 \times 10^{6}$ & 3.6 & 0 \\
\hline 80 & 0.95 & $88.8 \times 10^{6}$ & 8.8 & 0 \\
\hline 81 & 0.98 & $88.8 \times 10^{6}$ & 8.1 & 0 \\
\hline
\end{tabular}


comparing FC-68 -FC-69 -FC-70 with special attention to chord-wise pressure distribution along

butt-line 80. At the lowest Mach number, FC$68(\mathrm{M}=0.90)$, all the $\mathrm{Cp}$ values are subcritical, except for just at the leading edge where indeed there may be a shock. Downstream of this the comparison CFD with flight test is reasonable. At the next higher Mach number, FC-69 ( $\mathrm{M}=0.95)$, the supercritical flow region extends further downstream of the leading edge, it terminates in a shock, but CFD and flight test do not agree on the position. And at the highest Mach number, FC-70 ( $\mathrm{M}=0.97)$, the discrepancy increases.

Tomac $^{7}$ [2014] has studied this suite of flight conditions with RANS analysis and EARSM turbulence modeling with three distinct grids plus adaption along with associated aircraft modeling. Their first grid models the wing as a single solid element (as do all the other contributors with their grids). Their second and third grids model the three control surfaces (leading edge flap, trailing edge elevens and ailerons) with gaps (see Fig 12) where the surfaces are not deflected, and then where they are deflected down (by l.e. flap 3 deg, elevon 1 deg and aileron $2 \mathrm{deg}$ ). These three grids can also be solution-feature adapted by sensors that turn on at shock waves and vortices. Figure 16 shows an example of such adaption triply adapted) for the treating the shock-vortex interaction problem.

In addition, Jirasek ${ }^{15}$ [2014] took account of elastic effects of the aircraft by a firstorder aeroelastic analysis (see Aeroelastics in Section $\mathrm{V}$ below). This resulted in a fourth grid to include the elastic twist that was determined through the aeroelastic analysis, and is compared with the fifth grid, identical, but for the rigid aircraft.

Figure 17 plots the comparison of these five grid results on the inner wing section at BL70 and shows that except for small effects at the trailing edge gaps, surface deflections and aeroelastic twist have very little effect on the pressure distribution, and thus do not bear upon the discrepancy in shock position. However, on the outer wing, BL153 in Figure 18 we do see a more marked effect, although little correlation with the measurements.
Special Session on CAWAPI-2 Aerodynamics

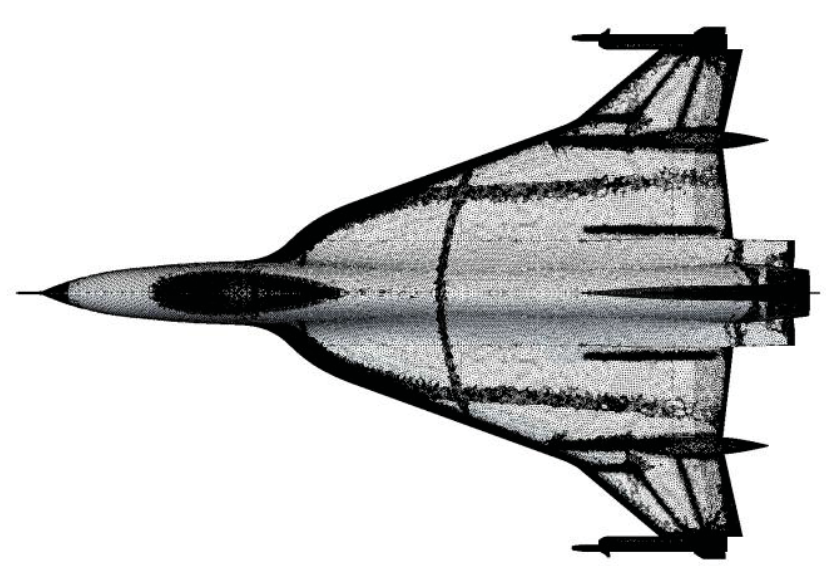

Figure 16. Tomac' [2014] grid triply adapted to the shockvortex interaction problem of FC-70. Control surfaces are modeled including gaps, zero deflections.
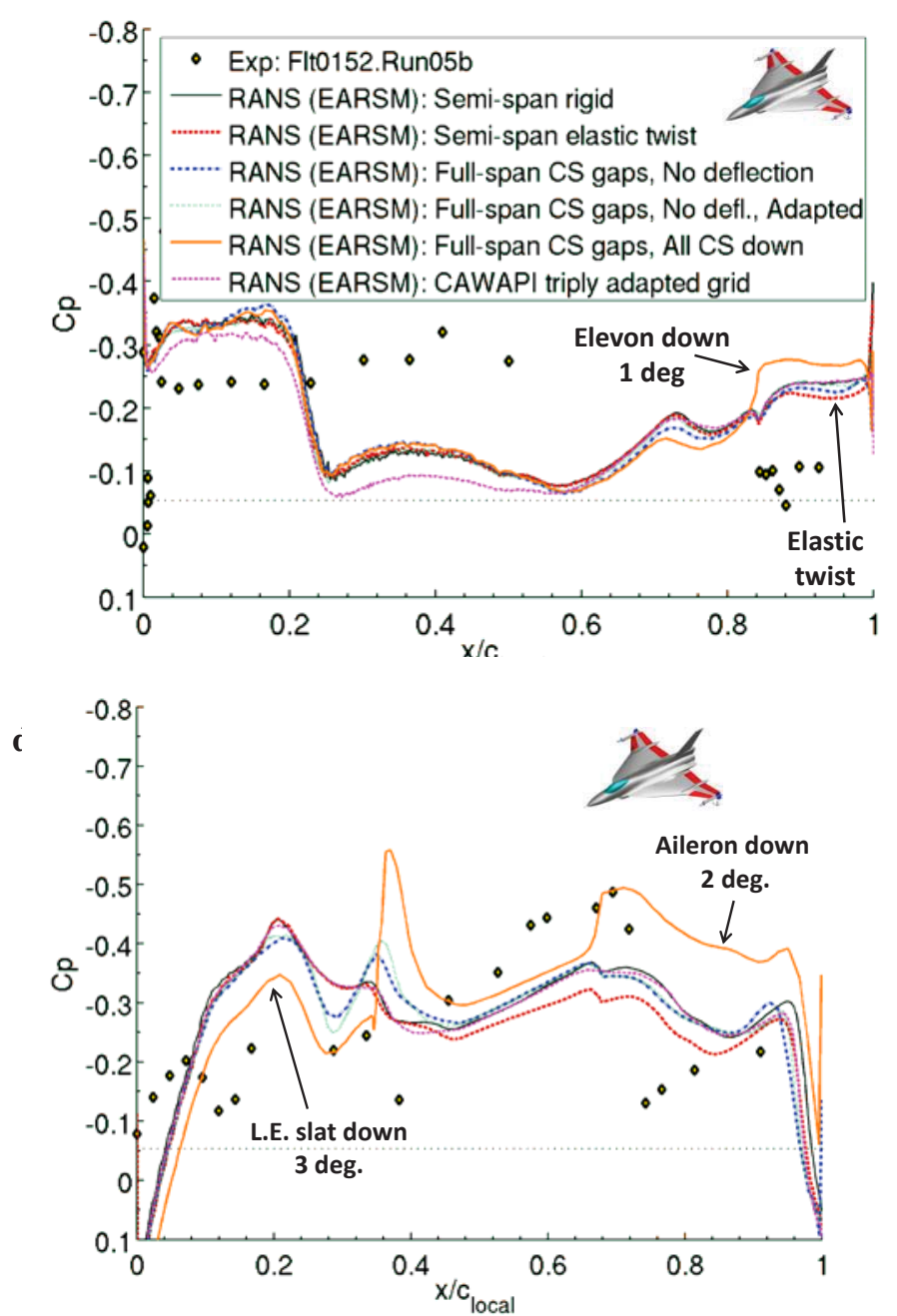

Figure 18. Tomac $^{7}$ [2014] comparison of chordwise Cp distributions for various grids. Outer wing, BL-153, FC-70. 


\section{B. Baseline Condition FC43}

In an attempt to understand how these factors influence surface pressure, the authors investigated the "mildest" condition FC-43, see Figure 19. They found that on the inner wing the $\mathrm{C}_{\mathrm{p}}$ compared reasonably well, except for some discrepancies at the start of the vortex. On the outer wing however, Figure 19 indicates a mismatch at the leading edge which possibly could be explained by deflecting the leading edge flap up instead of down. But this explanation must be verified by a new computation.

\section{Transonic Case at moderate alpha FC79}

It is possible that the difficulties encountered for FC-69 and FC-70 are associated with the very weak leading-edge vortex associated with the low angle of attack for these cases $\left(\alpha=5^{\circ}\right)$. In these circumstances the vortex resides very close to the wing boundary layer. It also may be sensitive to wing geometry details such as from the s-blend region of the wing leading edge.

Transonic vortical predictions were further assessed at a moderate angle of attack with FC79, and results are shown in Figure 20. The stronger vortex is clearly evident as are the stronger shocks from this surface pressure coefficient contour image. If nothing else, these vortices would be better grid resolved.

Correlation between CFD and flight test are shown in Figure 21 along Butt Line 40. At this inboard station the correlation is quite good, both for shock strength and shock location. Correlations at Butt Line 70 were less encouraging but still better than the previous prediction deficiencies from FC-70 and the CAWAPI predictions. Overall, this is encouraging progress for predicting these transonic, shock-vortex interaction aerodynamics.

\section{Modeling Assessment Summary}

Here we summarize, from both focus problems what we think we've learned. I guess this is also where we point out what is missing, what needs to be done, future work, etc.

\section{A. Flow Modelling}

\section{Better Grids.}

There has been a steady progression of grid resolution throughout the F-16XL computation assessments. Lamar's [2001] early work started with 1 million cells (semispan discretization). The CAWAPI program averaged 16 million cells, and now CAWAPI-2 has averaged 46

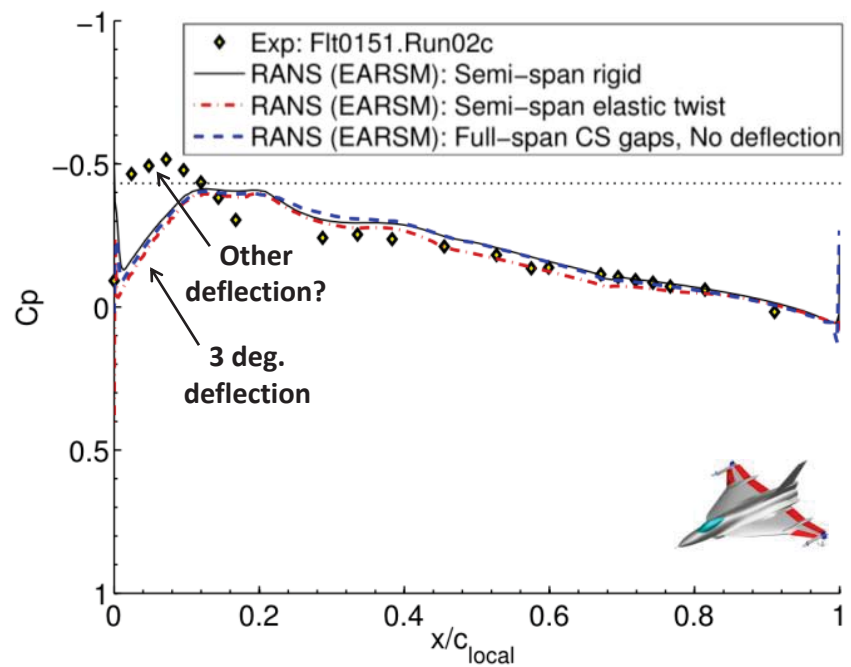

Figure 19. Tomac ${ }^{7}$ [2014] comparison of chordwise $C_{p}$ distributions for various grids indicating perhaps that l.e. flap deflected up instead of down. Outer wing, BL-153, FC-43, $M=0.81, R_{\text {cref }}=69.6 \times 10^{6}, \alpha=5^{0}$.

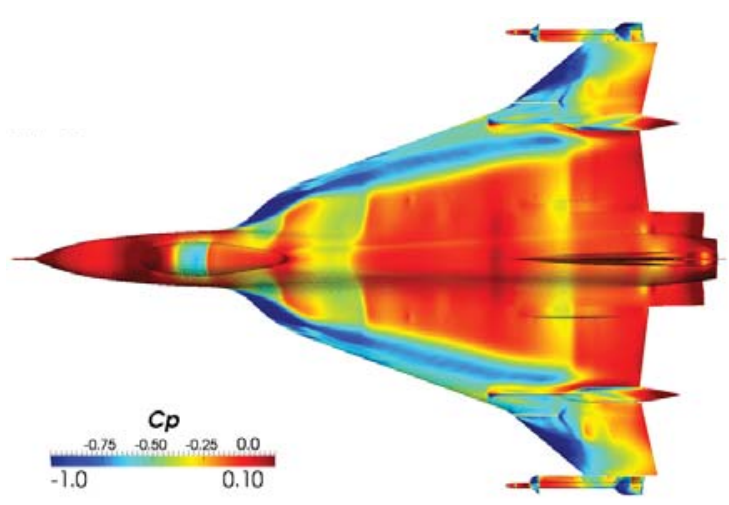

Figure 20. Transonic moderate angle of attack prediction. FC-79, $\mathrm{M}=0.90, \mathrm{R}_{\text {cref }}=89 \times 10^{6}, \alpha=9.3^{\circ}$

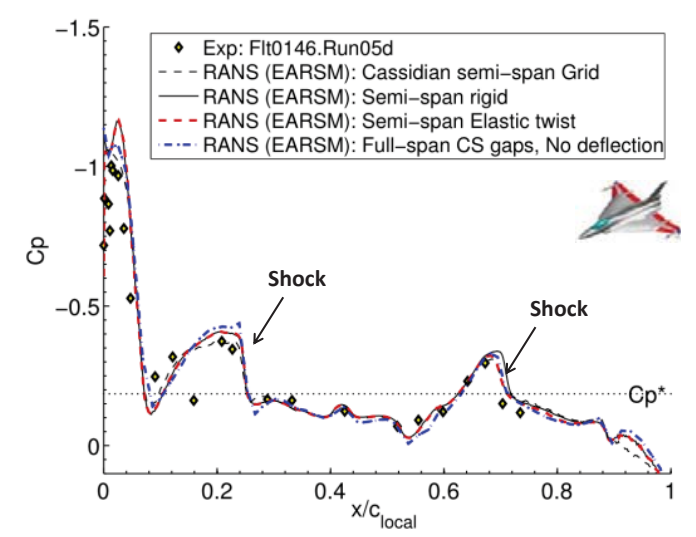

Figure 21. Transonic pressure coefficient prediction. FC-79, $M=0.90, R_{\text {cref }}=89 \times 10^{6}, \alpha=9.3^{\circ}$ 
million cells (with a maximum grid of 143 million cells). The larges grid was necessary for secondary vortex resolution [Elmiligui $\left.{ }^{13}, 2014\right]$ and is not uncommon, from the author's experience, for modeling flight vehicle geometries. Grid assessment studies with much large grids will be needed to determine conditions of grid convergence.

Grid adaptation was inconclusive for the subsonic application and improved the transonic application studies, Flows with vortices, shocks, and shock-vortex interactions have high flow-gradient regions in the flowfield near the vehicle but away from the wall-bounded flows, and for these flows an effective and reliable grid adaptation capability is still highly desirable. It does not appear that we have this capability at the time of this writing, and further research must be encouraged on this topic.

In the meantime, it must be commented that the targeted clustering Hitzel ${ }^{12}$ [2014] used for his vortex breakdown studies was very effective. (Some of this grid work was shown in Figure 7). The targeted clustering was a common sense approach that put high grid density in regions of the complex vortical flows at a total grid count of only 45 million points (semispan). This approach may be required for vortex breakdown studies since breakdown is an inherently unsteady phenomenon, and time-accurate adaptive grids do not appear to be forthcoming.

\section{Improved Physics}

Insight to the vortical and shocked flows for the two focus problems has been advanced through the CAWAPI-2 research. A physics-based approach the numerical studies has at least related trends observed among the numerical simulations to flow structures that occur on the F-16XL-1 vehicle at the target conditions of interest. However, the details of the flow physics, and their connections to the details of numerical models, have yet to be established. In many cases we now understand what we have, but most of those same cases it is not yet clear why we have such results. Some highlights follow.

For the low speed focus problem it has become clear that unsteady simulations pertinent to vortex breakdown are crucial to the outer panel simulations. Hitzel's work has focused on breakdown, and provided insights to its consequences for the F-16XL-1 outer panel aerodynamics. His work has pointed to the importance of simulating breakdown effects, and his results are very encouraging for the specific F-16XL-1 study. However, it must be also noted that proper modeling of vortex breakdown phenomenon is still an open question, and not one of the CAWAPI investigations. To the authors' knowledge there is no validated modeling established for CFD predictions of vortex

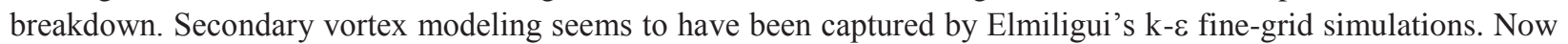
that we have seen this outcome, it would be instructive to understand the details of just why the simulation has produced such an outcome. The accomplishment is encouraging, but is it a general advancement or a particular fit to a particular case.

For the transonic speed focus even less certainty is in hand, due perhaps to more interacting effects of physics modeling combined with aircraft modeling. Grid adaption appears to improve the resolution of the shock-vortex interaction problem, but the computations still fail to predict its location on the inner wing, and further incorrect predictions occur on the outer wing. Effects due to physical modeling have not shown significant effect, so presumably the explanation does not lie there. Aeroelastics and control surface deflections (which we have little knowledge of from the flight testing) seem to become more important for this flight regime than for the subsonic regime. Some progress nonetheless has been made here, but much more is needed.

\section{B. Aircraft Modelling}

\section{Aeroelastics}

Jirasek $^{15}$ [2014] carried out a usual static aeroelasticity computation where the structure is represented by the modal solver; the damping is set to critical damping so the solution converges to the static aeroelastic solution. This analysis yielded the expected deflection of the airframe under the FC-70 flight condition. Once the wing deflection was determined the authors then manually twisted their RANS grid to account for the elastic deformation in twist. This was the modelling used here, and as such can be considered adequate for the purposes of CAWAPI-2. It did not include any bending and one can expect that the effect of bending on the aerodynamic forces is negligible. Doing so simplified the deformation of the Euler mesh in the aeroelastic analysis. Figure 22 presents some results from this analysis. Then, to avoid the issue of just looking at the Euler results from the aeroelastic procedure, which would bring questions of vortex onset, the aeroelastic deformation can be manually given to the RANS mesh for CFD use. 

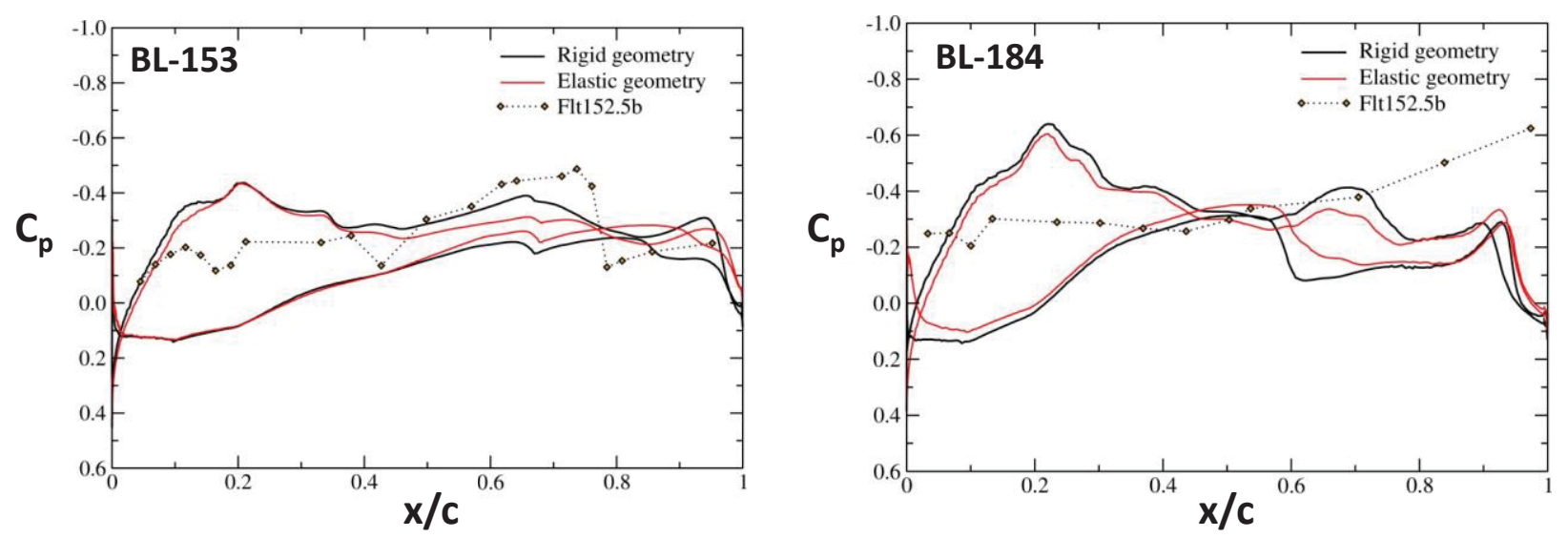

Figure 22. Pressures computed on rigid and elastic aircraft configuration for two Butt Lines. FC-70, $M=0.97, R_{\text {cref }}=89 \times 10^{6}, \alpha=4^{0}$.

Thus the aeroelastic analysis was done in two steps, first was to run the Euler computation of the CAWAPI aircraft including the aeroelastic model (structural modal representation). It started using a mesh built on a "baseline" model without any deformation which is assumed to be a jig shape. The result of this step is the aeroelastically deformed aircraft at flight condition FC-70. The final deformation is expressed as a summation of two modal shapes each multiplied by its generalized modal coordinate. The geometry deformation is rather large and deformation of the Navier-Stokes mesh was not successful. It was therefore decided to use only the twist deformation and assume that the bending does not have any effect on the aerodynamics of the wing. The final

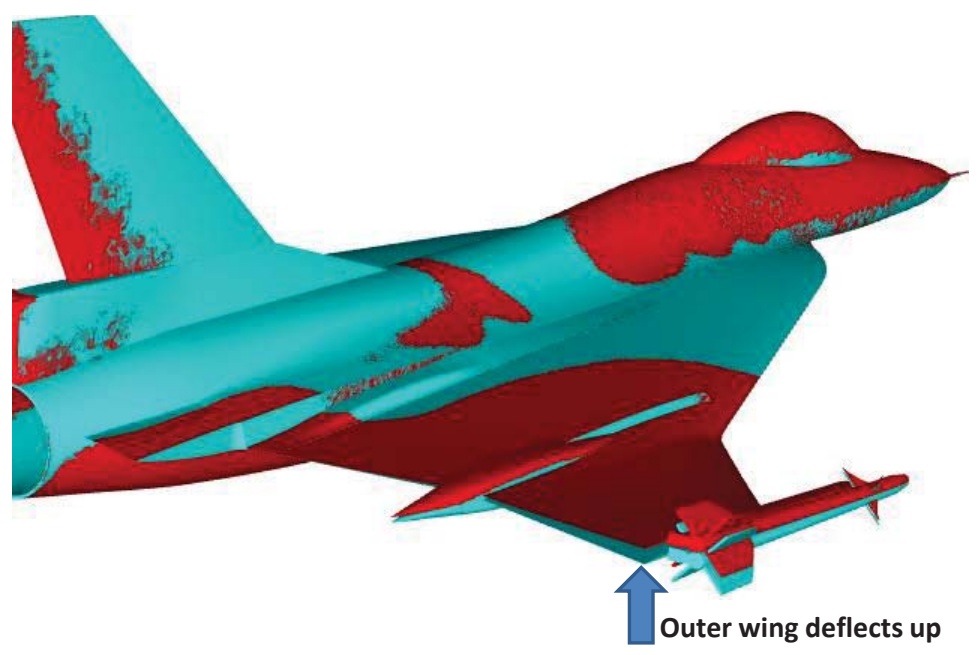

Figure 23. Rigid (blue) and aeroelastically deformed aircraft surface (red). FC-70, $M=0.97, R_{\text {cref }}=89 \times 10^{6}, \alpha=4^{0}$ deformed Navier-Stokes mesh is then used to analyze the aircraft aerodynamics and compare the results to the rigid aircraft. Figure 23 indicates the extent of the deflection given to the RANS grid model. The results can be compared to the inviscid solution on the rigid and elastic aircraft too, as a means of accuracy control.

\section{Control Surfaces}

Control surfaces are active continuously during flight testing. Not having them in our modeling leaves the door open to all sorts of sources of error and uncertainties in understanding. Even if the surfaces are not deflected, there are gaps, and presumably flow in the gaps. And such features can be likely causes for discrepancy when comparing results measured on a model with control surface (even just gap flow) with that computed on a solid surface model. Furthermore, we don't even know the geometry of the gaps or how the control surfaces are actually situated in relation to the core wing because the CAD description available to us only represents a solid one element wing. In addition this CAD description has been worked over by several contributors to make it water-tight, trim surfaces etc in order to create upon it a CFD grid (see Ref 3 for further details about this).

In order to get some kind of a handle on this open issue, Tomac ${ }^{7}$ [2014] has developed a surface geometry and associated computational grid that accounts for the three control surfaces of the F16-XL, the leading edge flaps, the elevens and the ailerons taking into account as much of the incomplete data that is available to us. The result is an aircraft model where the inner wing is a two-element geometry and the outer wing a three-element geometry where the control surface area predominates over the central core element. 
This is the first attempt to come to grips with this issue. And already what we have has indicated there are substantial effects, as large as or larger than physics modeling and grid resolution have produced. Results computed at low speed (FC-25) reveal marked rise in the peak in Cp under the vortex core at BL-80 as well as at BL-153, for instance. At transonic speed (FC-70) the effects are dramatic on the outer wing. This is just the start, and more development and application of this modeling is called for.

\section{Recommendations}

For the subsonic studies there was an effective division of study topics among the contributing authors, and each contributed advancements to their sub-study topic. It would be very useful to conceive and assess a best-practice approach to the subsonic problem based upon an amalgamation of the individual findings among the contributing researchers. Such a study would of necessity start with the subsonic high alpha focus problem. Any best practice approach would also need to be assessed for related flight conditions, perhaps some of the CAWAPI ones excluded from the CAWAPI-2 work.

Subsonic work also needs to be performed to better understand why the results came out as they did. Now that we have seen some new results that clearly are better than those from the prior CAWAPI studies, it is compelling to seek the physics-based reasoning between the phenomena of interest and the details of the particular numerical models employed.

The results presented indicate that discrepancies seen in the low speed cases vary in degree, but that those found for transonic cases vary not in degree but in kind. For example, the position of the shock that is computed differs so greatly from that what is computed and none of the modeling procedures tested so far provide enough authority over the solution to reduce the large discrepancy. Based on what we have seen so far, aircraft modeling would appear to offer more promise for progress than physical modeling. So, one recommendation is to continue with bringing the computational geometry model into better line with the flight test configuration. In the first place this means the control surfaces, and gaps. But the same could be said for controlling the geometry of ogive apex of the actual aircraft wing. The computational model is derived from a modified CAD file, but we don't know if the actual aircraft was manufactured form this file, or another. And even if it was, there are questions of manufacturing tolerances that can be raised. The suspicion is that the leading edge description may play a critical role for the onset of the rather weak vortex that lifts off the apex at low to moderate alpha and high speed. Alternatively, perhaps the discrepancy in kind warrants study in the wind tunnel where such factors are better controlled.

Finally, it is observed that many of the questions related to modeling the F16-XL flows could benefit from targeted wind tunnel tests. Flight-test data are invaluable for CFD assessments, but they also come with additional uncertainties as well as extreme costs for new data generation. Wind tunnel tests can be very cost effective for answering focused questions and to do so with quantified uncertainties. As a further benefit, such tests could be designed to provide insights for ground-to-flight scaling issues as they pertain to slender-wing vehicles.

\section{Concluding Remarks}

Considerable progress has been demonstrated by the computational project, CAWAPI-2. Specific improvements to flight test predictions have been associated with aerodynamic phenomena, such as vortex breakdown and secondary vortex flows, and other improvements have been found in association with aeroelastic deformation. The mesh density of these simulations also appears to have contributed to improved correlation with data, and has timeaccurate simulations. Further investigation into the details of these flows, and how those details are numerically manifested would be useful, and focused experimentation to guide this work would also be highly beneficial.

\section{Acknowledgements}

The authors gratefully acknowledge the support provided by their sponsoring organizations as well as the support provided to the individual researchers of this CAWAPI-2 effort. The NASA work was supported in part by the Fundamental Aeronautics Program, High-Speed Project. 


\section{References}

${ }^{1}$ Boelens, O. J., Badcock, K. J., Görtz, S., Morton, S., Fritz, W., Karman, S. L., Jr., Michal, T., and Lamar, J. E., “Description of the F-16XL Geometry and Computational Grids Used in CAWAP," Journal of Aircraft, Vol. 46, No. 2, 2009 , pp. 369-376.

${ }^{2}$ Boelens, O. J., Badcock, K. J., Elmiligui, A., Abdol-Hamid, K. S., and Massey, S. J., "Comparison of Measured and Block Structured Simulations for the F-16XL Aircraft,” Journal of Aircraft, Vol. 46, No. 2, 2009, pp. 377-384.

${ }^{3}$ Görtz, S., Jirásek, A., Morton, S. A., McDaniel, D. R., Cummings, R. M., Lamar, J. E., and Abdol-Hamid, K. S., "Standard Unstructured Grid Solutions for CAWAPI F-16XL,” Journal of Aircraft, Vol. 46, No. 2, 2009, pp. 385-408.

${ }^{4}$ Fritz, W., Davis, M. B., Karman, S. L., and Michal, T., "RANS Solutions for the CAWAPI F-16XL Using Different Hybrid Grids," Journal of Aircraft, Vol. 46, No. 2, 2009, pp. 409-422.

${ }^{5}$ Rizzi, A., Jirásek, A., Lamar, J. E., Crippa, S., Badcock, K. J., Boelens, O. J., "Lessons Learned from Numerical Simulations of the F-16XL Aircraft at Flight Conditions,” Journal of Aircraft, Vol. 46, No. 2, 2009, pp. 423-441.

${ }^{6}$ Morton, S. A., McDaniel, D. R., and Cummings, R. M., "F-16XL Unsteady Simulations for the CAWAPI Facet of RTO Task Group AVT-113,” 45th AIAA Aerospace Sciences Meeting and Exhibit, Reno, NV, AIAA Paper 2007-0493, Jan. 2007.

${ }^{7}$ Tomac, M. Jirásek, A., and Rizzi, A., "Factors Influencing Accurate Shock Vortex Interaction Prediction on F-16XL Aircraft," AIAA 2014-xxxx, Jan 2014.

${ }^{8}$ Le Moigne, Y., “Adaptive Mesh Refinement and Simulations of Unsteady Delta-Wing Aerodynamics,” Ph.D. Thesis, Royal Inst. Of Technology, Farkost och Flygteknik, Stockholm, 2004.

${ }^{9}$ Boelens, O.J., "CFD Analysis of the Flow Around the X-31 Aircraft at High Angle of Attack," AIAA 2009-3628, Jun 2009.

${ }^{10}$ Lamar, J. E., Obara, C. J., Fisher, B. D., and Fisher, D. F., "Flight, Wind-Tunnel, and Computational Fluid Dynamics Comparison for Cranked Arrow Wing (F-16XL-1) at Subsonic and Transonic Speeds," NASA TP 2001-210629, Feb. 2001.

${ }^{11}$ Boelens, O. J., "Flow Analysis of the F-16XL Aircraft (CAWAPI-2) at Transonic Flow Conditions," AIAA 2014-Xxxx, Jan 2014.

${ }^{12}$ Hitzel, S., "Vortex Flows of the F-16XL Configuration - CAWAPI-II Free-Flight Simulations," AIAA 2014-xxxx, Jan 2014.

${ }^{13}$ Elmiligui, A., Abdol-Hamid, K., Cavallo, P. A., and Parlette, E. B., "Vortex Numerical Simulations for the F-16XL Aircraft Configuration," AIAA 2014-xxxx, Jan 2014.

${ }^{14}$ Jirásek, A., Tomac, M., and Rizzi, A., "Improved Predictions for high Angle of Attack Flow around the F-16XL Aircraft," AIAA 2014-xxxx, Jan 2014.

${ }^{15}$ Jirásek, A., Cavagna, L., Riccobene, L., Ricci, S., and Rizzi, A., "Estimating first-order aeroelastic effects on F-16XL using NeoCASS/Edge analysis," AIAA 2014-xxxx, Jan 2014. 